\title{
Analysis of the Monomeric Friction Coefficient of Elastomers by Means of Dynamic Mechanical Properties
}

\author{
A.J. Marzocca, C.L. Matteo, J.J. Gonzalez* and R.B. Raimondo \\ Laboratorio de Propiedades Mecánicas de Polímeros y Materiales Compuestos, Dto. de Física, FCEN, \\ UBA, Pab. 1, Ciudad Universitaria, (1428) Buenos Aires, Argentina \\ * Electrical Engineering Department, Colorado State University, Fort Collins, CO 80523, U.S.A.
}

\begin{abstract}
The variation of the monomeric friction coefficient of styrene butadiene copolymer (SBR) and natural rubber (NR) with various degrees of cure was analyzed. Samples with different levels of cure were prepared. Loss tangent measurements of the samples were performed by means of a damped torsion pendulum between $173 \mathrm{~K}$ and $373 \mathrm{~K}$. Data were analyzed in the frame of the Kloczkowsky, Mark and Frisch model (KMF) and the monomeric friction coefficients and the relaxation strength were obtained for each condition.
\end{abstract}

\section{INTRODUCTION}

In 1990 Kloczkowsky, Mark and Frisch [1] presented a model based on earlier works [2,3] to explain the dynamic of polymer chains. They calculated the relaxation spectrum for tree-like networks of phantom Gaussian chains. The model proposes an extension of and idea from Graesley [3] by taking into account the bifunctional junctions dividing each chain between $\phi$-functional junctions into $n$-subchains of equal length. In this way, fractional interactions occurs not only at multifunctional junctions but also at bifunctional ones. Using this approach, the relaxation spectrum is considerably broadened and a more realistic description of the spectrum at shorter times is given.

Later, Heinrich [4] applied the KMF model to obtain the dependence of the loss tangent ( $\tan \delta$ ) on frequency of several elastomers using molecular parameters obtained from the literature. The model is applied in the short-time region identifying the junctions with the entanglement points.

To the best of our knowledge, the KMF model has not been fitted to experimental data of dynamic moduli of elastomers to obtain the molecular parameters as the primary relaxation time $\left(\tau_{0}\right)$, the relaxation intensity or the monomeric friction coefficient $(\zeta)$. In this paper we apply the KMF model to loss tangent data measured in SBR-1712 and NR in our laboratory. This study involves the analysis of the variation of the relaxation time and the monomeric friction coefficient with the degree of cure of the elastomer.

\section{THEORY}

In the KMF model, the network has the topology of a symmetrically grown tree where it is assumed that both $\phi$-functional and bifunctional junctions have the same frictional coefficient $\zeta$.

The motion of each junction is determined by the force balance

$$
-\zeta \frac{d\left\{r_{k}\right\}}{d t}-K \Gamma\left\{r_{k}\right\}+\left\{f_{k}\right\}=0
$$


where $\left\{r_{k}\right\}$ are the position $\bar{s}$ of the $N$ junctions, $K$ is the spring constant (as in the Rouse model [2]), $\Gamma$ is the connectivity matrix, and $\left\{f_{k}\right\}$ is a set of random, stochastic forces acting on the functions.

The relaxation spectrum is determined from the eigenvalues of the connectivity matrix, in the limit $N \rightarrow \infty$. The distribution of eigenvalues $\lambda(\xi)$ and the relaxation times $\tau(\xi)=\tau_{0} / \lambda(\xi)$, can be described by a continuous variable $\xi$, where $\tau_{0}$ is the primary relaxation time of a single unattached subchain, i.e. $\tau_{0}=\zeta / K$. Therefore, the relaxation spectrum is defined by

$$
H(\tau)=v k_{B} T \frac{d \xi}{d \ln \tau}
$$

where $v$ is the number of junctions per unit volume, $k_{B}$ the Boltzmann constant and $T$ is the temperature. Following a long but straightforward calculation [1], the relaxation spectrum is

$$
H(\tau)=\frac{v k_{B} T \tau_{o}}{2 \pi \tau} \frac{y^{\prime}(x)}{\sqrt{1-y^{2}(x)}}
$$

where $y(x)=\left[U_{n}(x)+(\phi-2) U_{n-1}(x)+(\phi-1) U_{n-2}(x)\right] /[2 \sqrt{\phi-1}], \quad x=1-\tau_{o} / 2 \tau$ and the function $U_{n}(x)$ is the Chebyshev polynomial of the second kind. The function $H$ is defined for the range of $x$ where $|y(x)|$ is less than 1 and $y^{\prime}(x)$ is positive; otherwise, it is taken as zero. The relaxation magnitude $\delta G$ is calculated from the relaxation spectrum as $\delta G=\int_{-\infty}^{\infty} H(\tau) d \ln \tau$. This value can be used to normalize the relaxation spectrum as $\Psi(\tau)=H(\tau) / \delta G$. Once the relaxation spectrum is known, the loss tangent can be easily calculated from the quotient between the imaginary $\left(G^{\prime \prime}\right)$ and real $\left(G^{\prime}\right)$ part of the complex modulus, that is

$$
\tan \delta=\frac{\int_{-\infty}^{\infty} \Psi(\tau) \frac{\omega \tau}{1+\omega^{2} \tau^{2}} d \ln \tau}{\frac{1}{\Delta}+\int_{-\infty}^{\infty} \Psi(\tau) \frac{\omega^{2} \tau^{2}}{1+\omega^{2} \tau^{2}} d \ln \tau}
$$

where $\omega$ is the circular frequency and $\Delta$ is the relaxation strength, defined as $\Delta=\delta G / G_{e}$, with $G_{e}$ the elastic modulus, that is the storage modulus at $\omega=0$. The influence of the temperature in eq.(3) can be introduced by using the WLF equation in the estimation of $\tau$ and $\Delta$ [5]. This method applies a shift factor $a_{T}$ to the measured frequency to relate loss tangent data which were measured at different temperatures. In fact, $\tan \delta\left(\omega a_{T}, T_{0}\right)=\tan \delta(\omega, T)$, that is, the loss tangent can be expressed, at a given reference temperature $T_{0}$, in terms of data measured at a different temperature $T$, but at a specific frequency related to the measured frequency as $\omega\left(T_{0}\right)=\omega(T) a_{T}$, since the shift factor $a_{T}$ is defined as the ratio of the relaxation time at temperature $T$ to that at the reference temperature $T_{0}$ [5].

\section{EXPERIMENTAL}

The rubber compounds were prepared with the composition given in Table 1 . The compounds were mixed in a laboratory mill. The molecular weight $M_{n}$ was determined by GPC as $174700 \mathrm{~g} / \mathrm{mol}$ for the NR compound (A) and $102400 \mathrm{~g} / \mathrm{mol}$ for the SBR compound (B).

Samples prepared as sheets of $150 \times 150 \times 2 \mathrm{~mm}$ were cured for different times at $414 \mathrm{~K}$ for NR and $433 \mathrm{~K}$ for SBR (Table 2). Samples were quenched in ice and water after being removed from the 
mold. For measurement of the dynamic properties, strip samples of $12 \times 2.8 \times 2 \mathrm{~mm}$ were cut using a die. Cure levels, $\alpha$, were estimated according to rheometer curves for each sample [6].

Dynamic properties were measured with a automated damped torsion pendulum in Ar atmosphere at 60 Torr [7] between $213 \mathrm{~K}$ and $373 \mathrm{~K}$ with a temperature ramp of $0.4 \mathrm{~K} / \mathrm{min}$. Frequencies between $0.1 \mathrm{~s}^{-1}$ and $20 \mathrm{~s}^{-1}$ were achieved applying different inertia moments to the pendulum. The maximum shear strain in the dynamic measurements was always less than 0.005 , thus ensuring linear viscoelastic behavior.

Table 1 - Compound Formulations

\begin{tabular}{|ccc|}
\hline & Compound A & Compound B \\
SMR20 & 90.4 & - \\
SBR 1712 & - & 89.3 \\
Zinc Oxide & 4.50 & 3.0 \\
Stearic Acid & 1.8 & 1.9 \\
Antioxidant & 0.9 & 0.8 \\
Paraffin & - & 1.6 \\
Processing aid & - & 1.3 \\
TBBS & 0.9 & 0.7 \\
TMTD & - & 0.2 \\
Sulfur & 1.3 & 1.2 \\
\hline
\end{tabular}

Table 2 - Sample cure data. $\alpha=$ cure level

\begin{tabular}{|cccc|}
\hline Sample & $\begin{array}{c}\text { Cure temperature } \\
\text { [K] }\end{array}$ & $\begin{array}{c}\text { Cure time } \\
\text { [min] }\end{array}$ & $\boldsymbol{\alpha}$ \\
A1 & 414 & 26 & 0.15 \\
A2 & 414 & 28 & 0.50 \\
A3 & 414 & 32 & 0.90 \\
A4 & 414 & 50 & 1.00 \\
A5 & 414 & 100 & 2.00 \\
B1 & 433 & 3 & 0.50 \\
B2 & 433 & 11.5 & 0.95 \\
\hline
\end{tabular}

\section{RESULTS AND DISCUSSION}

From the characteristic frequencies of the oscillations and the attenuation factors, measured in all the samples at different temperatures, the loss tangent was evaluated [7].

We developed a numerical program that fits eq.(3) to the data points and calculates the frequency shift factor $a_{T}$ [5] using the least squares method. In Fig. 1 and 2 the master curve at $298 \mathrm{~K}$ of $\tan \delta$ as function of the reduced frequency, $\omega a_{T}$, is shown for different cure levels of NR and SBR. It can be seen that there is a good agreement between the KMF model and our $\tan \delta$ data. The values of $\Delta$ and $\tau_{0}$ that optimize the fitting curve in each case are also calculated and are given in Table 3. It is interesting to note the influence of the cure level on the loss tangent. The curves are shifted to lower values on the frequency scale and a higher value of the relaxation time is obtained at higher cure levels.

Table 3 - Values of the parameters of the KMF model

\begin{tabular}{|ccccc|}
\hline Sample & $\log \tau_{\boldsymbol{o}}\left[\mathbf{s}^{-1}\right]$ & $\log \zeta_{\mathrm{o}}[\mathbf{N s} / \mathbf{m}]$ & $\Delta$ & $\tan \delta_{\max }$ \\
A1 & -8.05 & -9.84 & 14.83 & 0.745 \\
A2 & -7.99 & -9.78 & 9.93 & 0.662 \\
A3 & -7.03 & -8.83 & 9.96 & 0.650 \\
A4 & -6.60 & -8.40 & 8.02 & 0.622 \\
A5 & -6.85 & -8.65 & 6.47 & 0.576 \\
B1 & -7.03 & -9.25 & 69.19 & 0.959 \\
B2 & -6.78 & -9.00 & 32.10 & 0.915 \\
\hline
\end{tabular}

Once $\tau_{o}$ is calculated it is easy to obtain the value of the frictional coefficient of each monomer unit along the chain as $\zeta_{n}=12 k_{B} T \tau_{o} / z^{2} \ell_{s t}^{2}$ [1], where $\ell_{s f}$ is the statistical segment length and $z$ the number of monomers units within a statistical segment. In the case of NR $\ell_{s t}=0.88 \mathrm{~nm}$ and $\mathrm{z}=2$ [4], and for SBR $\ell_{s t}=1.06 \mathrm{~nm}$ and $\mathrm{z}=2.7$ [8]. The values of $\zeta_{\mathrm{o}}$ are given in Table 3 . It can be noted that $\zeta_{\mathrm{o}}$ increases at higher cure levels. In the case of slight cure level, the obtained values are similar to those given in literature for uncured NR and SBR [4]. The variation of $\zeta_{o}$ with the cure level is attributed to structural modification 
of the polymer when side reactions with sulfur take place in the molecular chain during vulcanization. A decrease of the maximum value of loss tangent is observed when the degree of cure of the elastomer is increased.

Finally, it should be remarked that the KMF model does not fit the experimental data of Fig. 1 at high frequencies, above the loss tangent peak, because it was developed for the rubbery and transition zone.

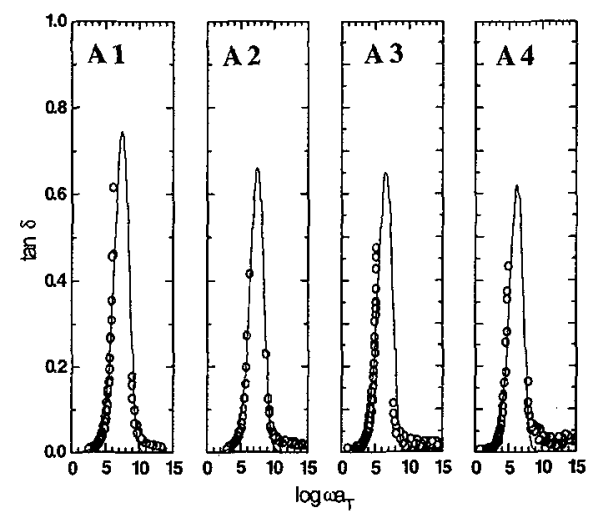

Fig. 1 Reduced loss tangent data for NR at different degree of cure. O Experimental data, - KMF model

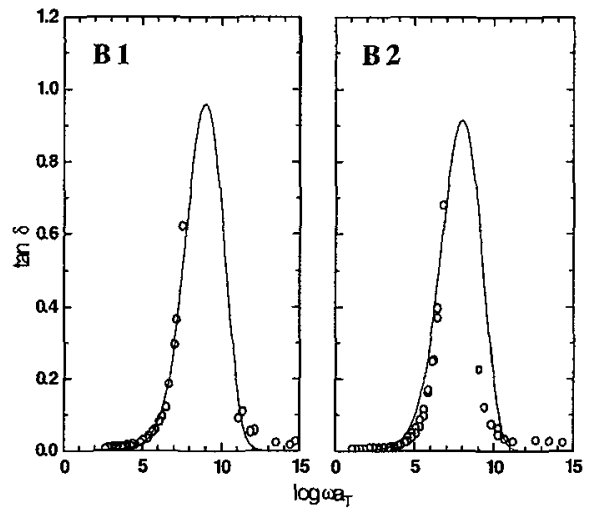

Fig. 2 Reduced loss tangent data for SBR at different degree of cure. O Experimental data, - KMF model

\section{CONCLUSIONS}

The variation of the monomeric friction coefficient in NR and SBR was evaluated from the KMF model fitted to reduced loss tangent data. Our results show that $\zeta_{0}$ increases with the cure level and, when the sample is overcured, $\zeta_{\omega}$ presents a slight decrease due to the degradation of the molecular structure.

\section{Acknowledgments}

This work was supported by the University of Buenos Aires, Argentina and the Fundación Antorchas (B.A.). The authors wants to thank Ms. G.Fernandez for the GPC measurements.

\section{References}

[1] Kloczkowski A., Mark J.E. and Frisch H.L., Macromolecules 23 (1990) 3481-3490.

[2] Rouse P.E., J.Chem.Phys. 21 (1953) 1272-1280.

[3] Graessley W.W., Macromolecules 13 (1980) 372.

[4] Heinrich G., Progr. Colloid Polym. Sci. 90 (1992) 16-26.

[5] Ferry J.D., Viscoelastic Properties of Polymers, (J.Wiley \& Sons, New York, 1980) pp 273-280.

[6] Hill D.A., Heat Transfer \& Vulcanization of Rubbers (Elsevier, London, 1971).

[7] Matteo C.L., Gonzalez J.J., Tischler J.G. and Marzocca A.J., Kaut. Gummi Kunstst. 48 (1995) 166172.

[8] Marzocca A.J., J. Appl. Polymer Sci. 58 (1995) 1839-1845. 